\title{
Differential resistance to distortion as a functional property of two structural parameters of two dimensional line drawings
}

\author{
R. F. COWAN AND W. D. BLISS1 \\ MONTANA STATE UNIVER SITY
}

Two structural parameters of two dimensional drawings, sidedness and regularity, are systematically varied and their relationship to the breakdown of binocular fuston (doubling of image) as a function of increasing lateral separation of dichoptically presented images is explored. Sidedness is not found to be systematically significant parameter, while regularity is seen to operate across figures (independently of sidedness). $A$ predictive analysis, in terms of the Gestalt variables of "good form" is examined, and the results discussed in terms of these variables, a personality dimension and uncertainty.

Two basic considerations are central to the development of a psychophysics of form. The first of these concerns the development of an index, or set of indices, that will completely describe the manner in which a shape is distributed in space. This is, of course, nothing more than a summary statement of the interaction between the underlying structural relationships and the compounded elements of a form which render it that which it is. The important word here is interaction, for in all instances, what we are attempting to predict is a functional property of form (whether that be aesthetic comparison or comparative recognition thresholds) from the interaction of its structural properties. In most instances, the structural relationships extent in a given form are readily discernible. The existing literature indicates that the plethora of physical measures which may be taken on the structural relationships of a simple, two dimensional black and white form are limited only by the ingenuity of the particular investigator. The comparative character of the functional properties of a form which arise from the interaction of these structural parameters, however, is considerably more difficult to specify. In most instances, these parameters will be moving in different directions of magnitude as one goes from form to form in any orderly progression.

The most complete statement of the interaction of the structural properties of a form is given in the form itself. It would further seem that any functional property of a form that can be demonstrated to differentiate that form from other forms of differing structural parameters would represent an adequate statement of the differential interaction of these parameters.

This brings us to a second consideration in the development of a psychophysics of form-that is, the identification of a functional property common in all forms to a greater or less extent. It is with this project that this study is concerned.

An enduring functional property ascribed to forms is that they are stable, hence, resistant to distortion. The studies in the literature have all measured this property in some fashion, though this has not always been the stated purpose. The method employed in these studies, however, has generally left the form percept intact while measuring response on some independent variable across a selective sample of forms (i.e., identification, associative response, recognition threshold, reproduction, scaled ratings, etc.). Two studies have attempted to test the strength of the percept through physical distortion of the stimulus target (Sander, 1928; Cowan \& Bliss, 1967).

The experiment undertaken here proposed to study the resistance of two dimensional line drawings to perceptual distortion induced through lateral separation of dichoptically presented targets to a point of fusional breakdown (doubling of image). Reasoning from the standpoint that resistance to distortion (of the type employed in this study) is a functional property which all forms possess to a greater or lesser extent as a function of differential structural properties, it was determined to examine two of these properties (number of sides and degree of regularity) in a two factor mixed model design with both factors completely crossed. The null hypothesis is simply that the stimuli employed in this study represent geometric forms drawn from the same population, and, as such, could not be expected to differ significantly from one another in terms of the dependent variable as a function of the structural parameters under consideration. Correlatively, the alternative hypothesis is that the differential structural parameters of these forms place them in different populations which can be differentiated among on the basis of their resistance to distortion effected through target divergence.

The foregoing hypothesis is seen to be nondirectional in terms of the structural parameters varied in this study. This obtains from the fact that as the number of sides of a regular polygon are successively increased, the relationships obtaining between these structural elements (symmetry, continuity, simplicity, balance, etc.) traditionally considered to reflect "good figure" are both increasing and decreasing. Thus, as sides are progressively increased, radial symmetry increases. If the "goodness" of a figure is a function of its degree of radial symmetry, then the more radially symmetrical figure should be the "better" figure. Further, if the functional stability of a figure is related to its "goodness" (Koffka, 1935) then the "better" a figure, the greater its functional stability and the more resistant to distortion (Cowan \& Bliss, 1967) it should be. This same argument, however, may be applied to the other relationships obtaining between the same structural elements of a form. Hence, if simplicity be conceived in terms of the number of elements involved in a figural production (i.e., line segments, angles, etc.), then the progressive addition of sides to a regular polygon results in the production of progressively more complex figures. If the "good" figure is the more simple figure, then one must expect the addition of sides to a regular polygon to produce progressively "poorer" figures up to some point of indistinguishability between the $n$ sided polygon and the most elementally simple figure, the circle.

We can see, thus far, that adding sides to a regular polygon has the effect of producing a symmetrically "better" form while simultaneously producing a "poorer" form in terms of simplicity. If the argument be extended to the concept of balance and this dimension is conceived in terms of equality of opposing sides and angles, then the progressive addition of sides to a regular polygon can be seen to alternately produce "good" and "poor" forms as one moves from odd to even sided polygons. The question becomes compounded into one not only of simple balance vs imbalance, but also of whether a balanced form is "better" or "poorer" than an unbalanced form of greater or lesser number of sides, and, hence, "better" and "poorer" symmetry and simplicity

The task, then, of specifying a directional hypothesis is seen to be, if not impossible, at least not intuitively simple; nor, is there precedent in literature for rendering such an hypothesis. For, if we identify the functional stability of a form with its "goodness," then we must predict that the "better" form will be more functionally stable, and more resistant to the type of distortion employed in this study. With respect to progressively increasing the number of sides of a regular polygon, however, we have just seen that there would appear to be no way of analyzing the functional interaction of relationships obtaining among the structural parameters of the form so as to specify its "goodness." Our best prediction, then, can only be that there is likely to be no straight forward, monotonic relationship between the number of 

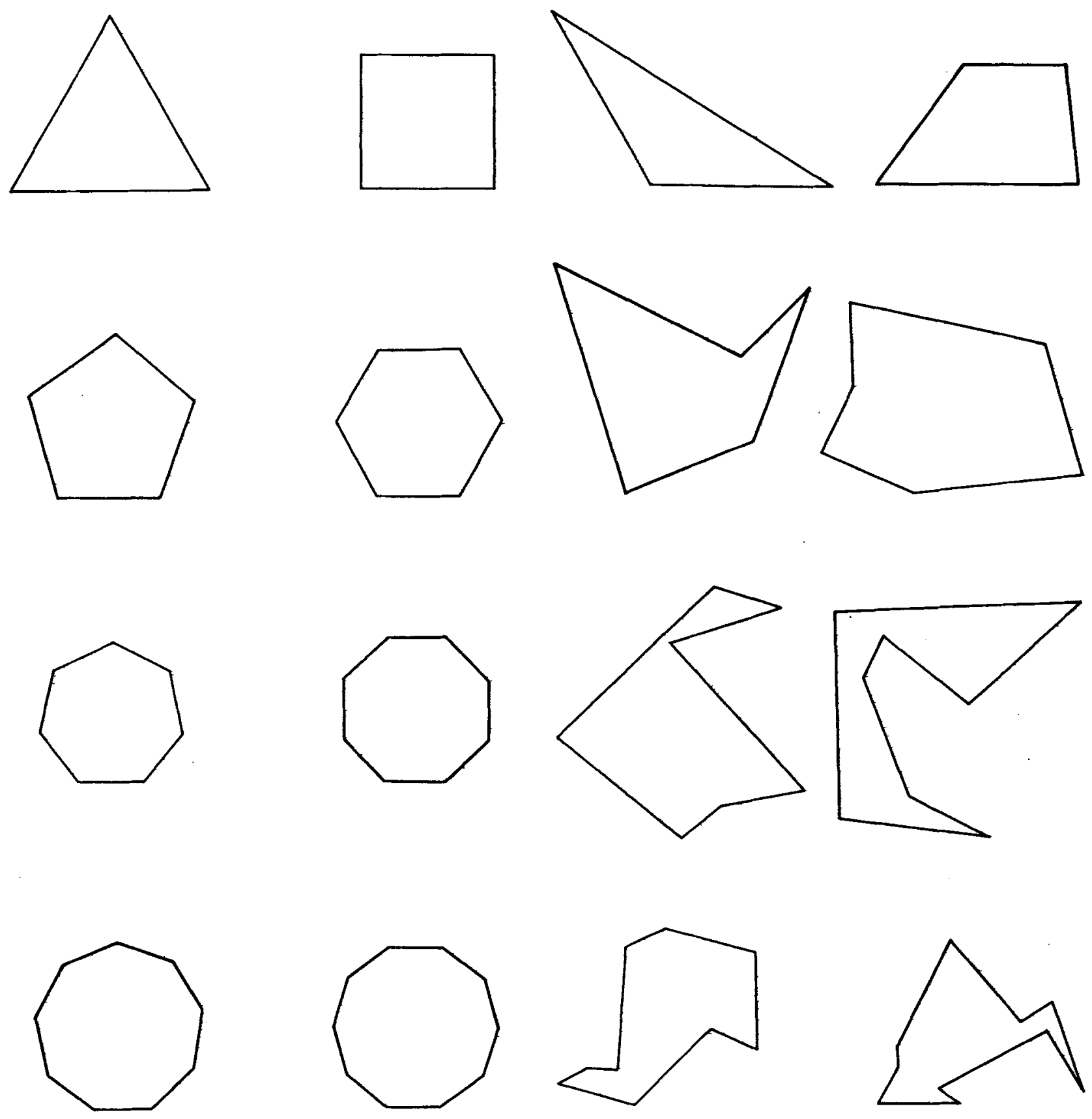

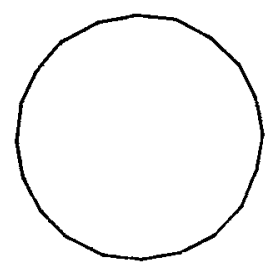

Regular Polygons

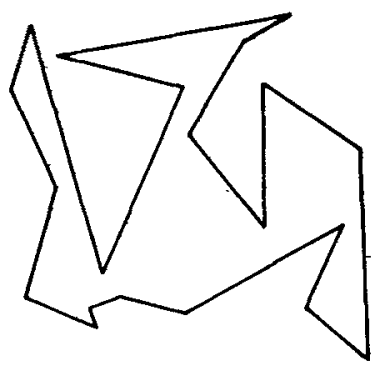

Irregular Polygons

Fig. 1. Stimulus materials. 
sides of a figure and its "goodness" expressed as diopteric resistance to distortion through lateral divergence.

With respect to regularity, since the completely irregular form has no radial symmetry, poor balance and indeterminate complexity, we might expect it to be the "poorer" form and a functional reduction of resistance to distortion to obtain as one moved from regular to irregular polygons. Again, however, the possible interaction of sides with regularity renders straight forward prediction impractical. Hence, our best estimate at this point must be a simple, non-directional hypothesis.

\section{Method}

Apparatus. A Renshaw Stereo-Disparator model of the standard Wheatstone Stereoscope was used to present stimulus materials. This instrument permits presentation of individual stimulus targets in each monocular field, which are experienced by the viewer as a single, fused target in a binocular field $87 \mathrm{~mm}$ on a side. Fusion strength, or resistance to distortion, may then be tested by separating the targets laterally from the vertical meridian, forcing the eyes to greater divergence until the fused image breaks down (doubles) in whole, or in part. The target holder for this study was set at $20 \mathrm{~cm}$ distance from the lens, which for this instrument-employing two base out +5 diopter lenses-represents a viewing distance of infinity. With the target holder at this setting, the demand upon accomodation is zero, so that a pure measure of convergence power may be calculated in diopters from the millimeter scale gauging lateral target separation. Target separation was set initially at zero so that each target fell directly in the line of visual regard for each eye separately thereby placing no demand upon convergence. All targets were viewed under normal room illumination.

Stimulus materials. Eighteen black line drawings of nine regular and nine irregular polygons were effected in duplicate on white stimulus targets $87 \mathrm{~mm}$ on a side. Each stimulus group (regular and irregular) was thus composed of two line drawings (on separate stimulus targets) of each of nine polygons (see Fig. 1). Each stimulus group comprised polygons of three through 10 sides plus one 20-sided figure. Stimulus forms were geometrically centered on targets so that when each stimulus pair was presented to the two eyes independently, corresponding points of each target stimulated corresponding points on the two retinas. Area was held approximately constant across all forms at $1600 \mathrm{sq} \mathrm{mm}$. Angles and side lengths (exclusive of the last angle and side length) for the irregular group of polygons were randomly generated by spinning a circular protractor over a pointer, and by a table of random numbers constrained in range to the limits of the targets dimensions. All Ss were presented each target pair in the same orientation.

There are six structural parameters of a polygon which may be varied--that is, total number of sides, regularity, size (area), rotation, dimensionality, and angularity. Of these, four were held constant or permitted to vary randomly, while two were varied systematically. The two varied systematically were number of sides and regularity, both in a fixed manner. It was determined to hold area constant since the latitude of variation was relatively constrained by the target dimensions. Rotation was not seen to be a differential variable in this study (as it might be in studies involving reaction time or recognition thresholds) since the nature of the task demanded fixation and scanning of targets for extended periods of time. Hence, all targets were presented in identical orientation. Dimensionality is an illusory effect which, perhaps, demands study in itself. In this task, however, its effect is to add another dimension of target complexity beyond the level of concern, and was, thus, held constant at two dimensions. Angularity, for completely regular polygons of fixed area is itself fixed. It was not, however, a dimension of concern in this study, and was permitted to vary randomly across irregular polygons of increasing total number of sides.

Subjects. A subject pool was first obtained from students in two sections of an undergraduate perception course. These students had no prior acquaintance with studies of this nature. From this subject pool, $20 \mathrm{Ss}$ were selected and matching each with the other on the basis of visual acuity (20/20 Snellen conversion), and comparability of lateral and vertical phoria (representing the middle 66 and $50 \%$ of the normal population respectively). These visual characteristics were obtained by testing each S on a Bausch \& Lomb Modified Ortho-Rater.

Procedure. Ss entered the testing room one at a time and were seated at the table upon which the test apparatus was situated. The viewpiece of the test apparatus was then adjusted to the $S$ for comfortable viewing. Each $S$ was told that he would be presented with two identical stimulus targets which when viewed through the stereoscope would be visible as a single fused image. He was instructed to then separate the targets laterally by turning a separator knob controlling the focal plane of the target holder until he reached a point where the fused target image broke down. Each $\mathrm{S}$ was told that he might experience transitory breakdown of the fused image in whole, or in part, followed by re-fusion; but, that he was to continue separation of the targets to the point where he could no longer maintain target fusion in whole, or in part. Ss were then given two practice trials with targets similar to, but different in dimensions and structure from the stimulus series. In each instance, Ss reported seeing a single fused image initially, followed by doubling at some point of lateral separation. During the administration of the crucial series, after each target pair was presented and the $S$ had separated them to the point of image doubling, he looked away from the viewer and relaxed his eyes while note was made of the degree of separation, the target holder returned to its initial setting and two new targets inserted. The procedure was then repeated for each of 18 pairs of stimulus targets. Target presentation was completely counterbalanced across all Ss after the method of Benjamin (1962) to control for fatigue, practice and response bias effects. This method permits differentiation and non-repetition of each series order.

\section{Results}

The mean resistance to distortion (as measured by the degree of lateral separation between targets of each monocular field at the point of fusional breakdown) for all target patterns in each group (regular and irregular) are presented in Table 1. These means reflect the distortion level pooled across 20 Ss under each stimulus condition. The total range of resistance to distortion in diopters varied from 0 to 15 . Analysis of variance by the mixed model design yielded a nonsignificant $F$ ratio for the variable of increasing number of sides. The graphic representation of this variable in Fig. 2 reveals a nonmonotonic quartic trend across sides as would be expected from the initial discussion. The regularity variable-that is, completely regular vs completely irregular forms produced an $F$ ratio significant at the .05 level $(F=3.57, \mathrm{df}=1)$, however the direction of this significance is toward greater resistance to distortion for the irregular figure (with the exception of Fig. 7) which is some what contrary to the expected results. The other effects were not significant.

\section{Discussion and Conclusions}

There are three results emergent in this study which are of primary interest. The first of these is the relatively large error term $(\mathrm{MS}=13.15, \mathrm{df}=342)$. Even though $\mathrm{Ss}$ were matched for comparability of visual system, it would appear that inter-S differences remain quite large. An examination of the data, $S$ by $S$, indicates a tendency for some Ss to respond with high levels of convergence $^{2}$ power across figures, while others respond with comparatively low levels. This could reflect a stable, systematic response style brought to the experiment by the $\mathrm{S}$. We are thinking here of something on the order of a major personality

Table 1 Mean Diopters

$$
\begin{array}{lllllllll}
\mathrm{A}_{3} & \mathrm{~A}_{4} & \mathrm{~A}_{5} & \mathrm{~A}_{6} & \mathrm{~A}_{7} & \mathrm{~A}_{8} & \mathrm{~A}_{9} & \mathrm{~A}_{10} & \mathrm{~A}_{20}
\end{array}
$$

$\begin{array}{lllllllllll}\text { Regular } B_{1} & 5.25 & 6.14 & 6.60 & 6.65 & 6.15 & 6.05 & 7.35 & 6.35 & 6.80\end{array}$ $\begin{array}{lllllllllll}\text { Irregular } & B_{2} & 7.10 & 7.10 & 7.50 & 7.70 & 6.75 & 6.45 & 6.80 & 7.30 & 7.75\end{array}$ 


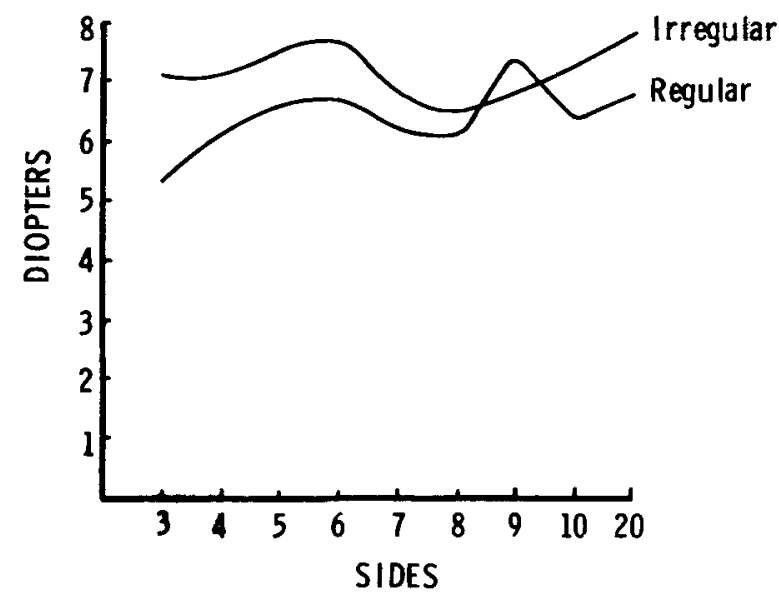

Fig. 2.

variable such as Witkin's et al (1954) "field dependent-field independent" perceivers, or Rotter's (1966) "high external control-low external control" variable. If this is indeed the case, what is now necessary is a study relating groups dichotomized on such variables to the tendency to exhibit high and low convergence scores.

A second feature of interest here is the significant effect of degree of regularity on the convergence eliciting power of the s timulus. The effect of going from completely regular to completely irregular forms is an increase in the "apparent" resistance to distortion of the figure. This would seem to imply, on the basis of Cowan and Bliss' (1967) results, that the irregular figure possesses more strength and is, thus, the "better" figure. If we conceive, rather, that the irregular figure is one of the higher uncertainty (either in the informational sense or the conventional sense), then it can be seen that the $S$, when presented with the irregular figure, is faced with less surety regarding its structural elements (and their behavior) relative to its regular counterpart. The effect, then, would be to render the $S$ less certain of the exact point of fusion (in part, rather than whole, most probably) breakdown took place such that he would in fact raise his reporting criterion to effect greater surety of his percept. This is to say, that since there is less certainty inherent in the stimulus object, the $S$ has less surety of its functional properties, and, consequently requires a stronger "effect" (relative to the regular figure) before reporting. The result is to drive the resistance to distortion measure up, which measure in this case is not measuring pure distortion resistance, but, rather, a compound of convergence pull and uncertainty. We cannot be certain, then, whether the significant effect obtained for this variable is strictly one of regularity or an interaction of $S$ variables with uncertainty inherent in the more irregular figure. What is now required, in this case, is to generate a population of irregular figures of known informational content, which (in a progressive series) will permit a study of the effect of uncertainty on convergence power. By utilizing a signal detection model, it would then be possible to separate the effects obtaining from an interaction of $S$ with uncertainty, and those due solely to the physical properties of the stimulus (here it is conceived that the structural parameters of form generate $S$ reactions other than strictly uncertainty).

The third feature of interest arising in this study is the absence of significant effects due to sidedness of the form $(F=1.59$, $\mathrm{df}=8$ ). Though the $\mathrm{F}$ ratio for this variable is insignificant, study of the curves shown in Fig. 2 indicates that the number of sides a figure possesses does in fact influence its convergence pull, and does so evenly across the variable of regularity, but not in any straight forward manner. As can be seen, there is a steady increase in convergence pull as one moves across figures from 3 to 6 sides, followed by a drop across 7 and 8 sided figures, and a subsequent increase to the pre-drop level for figures of 9,10 and 20 sides. It is doubtful, at least for the regular polygon, if this increase would be furthered beyond the 20 sided figure, since, as can be seen from Fig. 1, this form at this size is relatively indistinguishable from a circle. It is not, thus, sufficient to read off the factor of sides as insignificant, but, rather, to state that its significance is relatively enigmatic, particularly in so far as it appears to be independent of regularity and perceiver set. The prospect of meaningfulness of form configuration is, of course, influenced by sidedness. Again, however, such influence is not straightforward. For regular forms, at least, and in terms of cultural significance, it is difficult to understand why 7 and 8 sided figures should be different from 6 and 10 sided figures which do not differ greatly from one another. For this study, then, the influence of number of sides on convergence pull is, at best, abstruse.

From the discussion above, we can conclude that there may indeed be a significant effect operative in form perception as a function of perceiver "style." Further, that while degree of regularity is a significant variable in form perception, its effect, on this task, may be confounded with the uncontrolled variable of uncertainty, producing thereby an inflated convergence pull. Sidedness, while nonsignificant in this study, none the less appears to be an operative variable which is relatively independent both of regularity and perceiver "style."

\section{REFERENCES}

ALIMENA, B. S, A method of determining unbiased distribution in the Latin Square. Psychometrika, 1962, 27, 315-318.

COWAN, R. F., \& BLISS, W. D. Resistance to distortion as a metric for pattern goodness. Psychon. Sci, 1967, 9, 481-482.

KOFFKA, K. Principles of Gestalt psychology. New York: Harcourt Brace, 1935.

SANDERS, F. Beitrage zur Psychologie des stereoskopischen Sehens. I. Mittielung. Gemein sum mit Ryuon Jinuma, Formosa. Die Gunzel der binokularen Verschmelzung in ihrer Abhangigkeit vs der Gestalthohe der Dappelbilder. Arch. f. d. ges. Psychol., 1928, 65, 191-206.

ROTTER, J. B. Generalized expectancies for internal vs. external control of reinforcement. Psychol. Monogr., 1966, 80, 1-28.

WITKIN, H., LEWIS, H. B., HERTZMAN, M., MACHOVER, K., MEISSNER, P. B., \& WAPNER, S. Personality through perception. New York: Harper, 1954.

\section{NOTES}

1. Address: Department of Psychology, Montana State University, Bozeman, Montana 59715.

2. Convergence power is used when speaking from the perceiver orientation, and resistance to distortion, or convergence pull, when speaking from the stimulus orientation.

(Accepted for publication June 24, 1968.) 